\title{
Globalización, Fragmentación, Descentramiento y Construcción de Nuevas Identidades
}

Rafael DEL VILLAR

1. Intratución

El dossier de Nuestra Revista Comunicación y Medios No 13, año 13 tiene como núdeo temático la descripción semiótica de los procesos de "Globalización, fragmentación, decentramiento, y oonstruoción de nuevas identidades", problemátca del III Encuentro Chileno de Semiórica, realizado en Santizgo de Chile, los dias 910 de Abril 2001 . Este dossies, reproduce parcialmente las contribuciones desarroladas en dicho evento.

El III Encuentro Chileno de Semiótica fue organizado por la Asaciación Chilena de Semiótica, y el Deparamento de Investigaciones Medí́ticas y de la Comunicación, Escuela de Periodismo y Programa de Magister en Comunicación Social, de le Universidad de Chite. Contó con el auspicio de la Secretarla de Conanicación y Culura, del Gobierno de Chile; y el Departamento de Extensión Cultural de L Universidad de Chile; y la colaboración del Programa Ecosf Conicyt (Proyecro C00H01), que permitio financiar la verida de los Invitados extranjeros, de la Université de Paris III.

La temdicica del III Encuentro ha sido la descripción semiótica de los procesas de "Globalización, fragmentación, descentramiento, y la construbción de nuevas identidades", lo que ha implicado una base

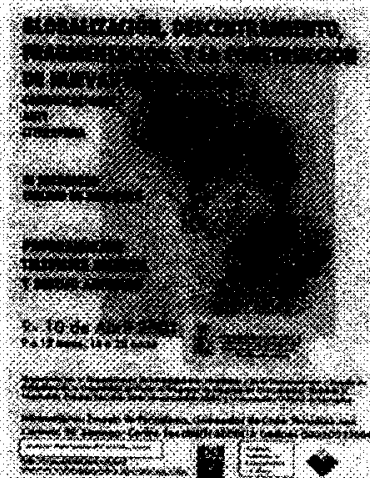


común, una matriz, que articula a todas las comunicaciones del evento; cryo objetivo final es producir una contribución a la inteligibilización de los nuevos procesos de construcción de identidades emergentes.

2 Globalización, descentramiento, fragmentación

Macro procesos de Transformación:

El desplazamiento de la comunicación pública a la comunicación en la vida cotidiana, como eje de referencia simbólico hegemónica, ha constituido una ruptura de funcionamiento de todo el edificio sociocultural, lo que ha sido descrito por diferentes autores, constituyéndose un saber adquirido al respecto. Dentro del pensamiento latinoamericano cabe destacar los análisis de Néstor García Canclini y Jesús Martín-Barbero y los diagnósticos al respecto constituyen ya una base respecto a la descripción de los macro- procesos de funcionamiento sociocultural producidos por la globalización y la diversificación cultural.

La caída del muro de Berlín, contribuirá al desaparecimiento de los dos polos de funcionamiento ideológicos que dividieron las cosmovisiones del siglo $\mathrm{xx}$, y que fueron la referencia simbólica de la totalidad societal.

El paralelismo de este proceso con la emergencia de las nuevas tecnologías comunicacionales, como la comunicación satelital, tv cable, videocassete, videojuego, control remoto, multimedia, internet, y el abaratamiento de los costos de la producción audiovisual, entre otros, multiplican la producción de la diversificación cultural. Lejos de homogeneizar las propuestas culturales, la globalización ha significado un aumento de la diversidad de oferta audiovisual. Ya en 1997 las investigaciones de Carlos Catalán y Alejandra Ramm describen que en el caso chileno la globalización ha significado un aumento de la producción nacional: "en 1982 la programación de origen nacional sólo alcanzaba el 39\% de la programación total, en 1996 el 56\% es programación nacional" (Catalán, Ramm, 1997: pág. 98).

El abaratamiento de los costos de la producción audiovisual va a tener, también, un correlato en el abaratamiento de los equipos, lo que implicará una expansión del equipamiento de los consumidores.

Estadísticas del Consejo Nacional de Televisión señalan que en Chile (Informe 1999): a) en los estratos socioeconómicos más pobres hay un 1,75\% de televiso-

14| res por hogar, esto es, de 3 familias a lo menos 2 tienen 2 televisores; y en los estratos altos hay 3 relevisores por familia; b) la presencia de videograbadores en los estratos populares es de un $32,1 \%$, en los segmentos medios de un $63,5 \%$, yen los altos de un $80,0 \%$; c) los video-juegos penetran en todos los estratos en forma más o menos similar: un $34 \%$ en los segmentos altos, un $24,3 \%$ en los medios, y en un $21,3 \%$ en 
los bajos, d) la inserción de la televisión pagada es de un $69,9 \%$ en los estratos altos, de un $45,0 \%$ en los medios, y de un 18,1 en los estratos populares; e) el 72,4\% del estrato alto y medio alto tienen cotnquador, un $50,6 \%$ del medio-modio tambien bo tienen, y un 2,0\% en los estratos más pobres.
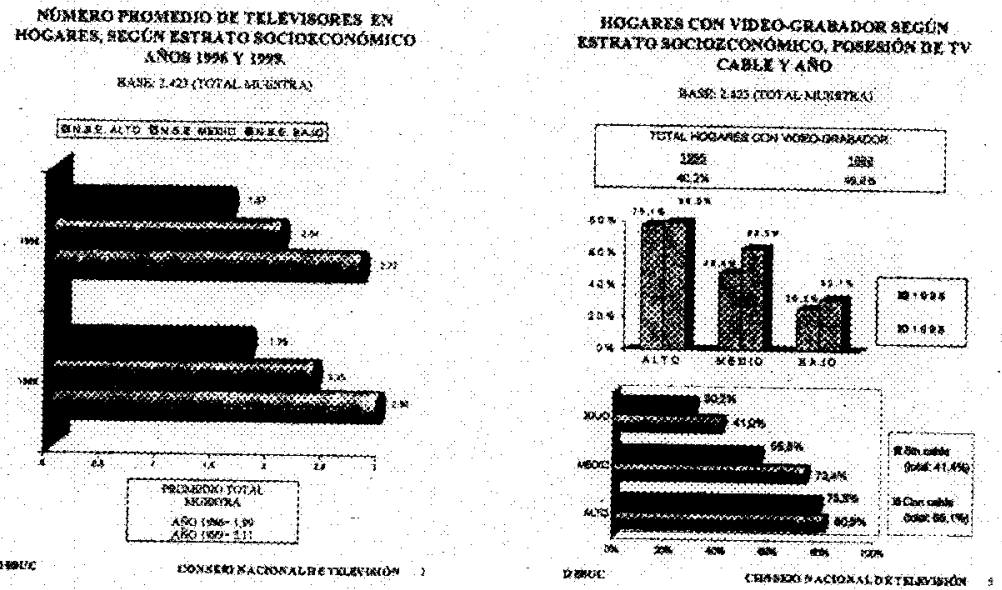

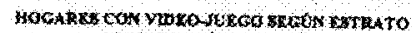

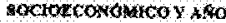

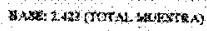
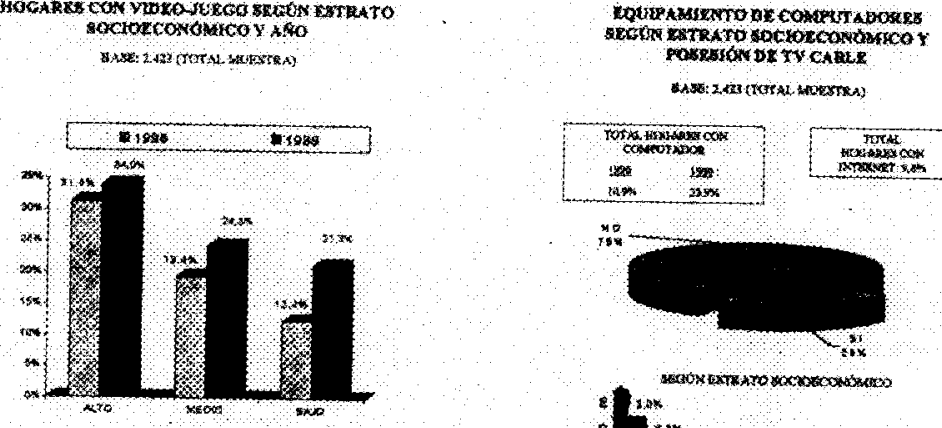

- 100
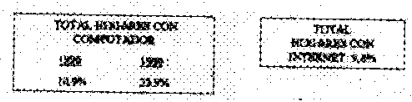

sis
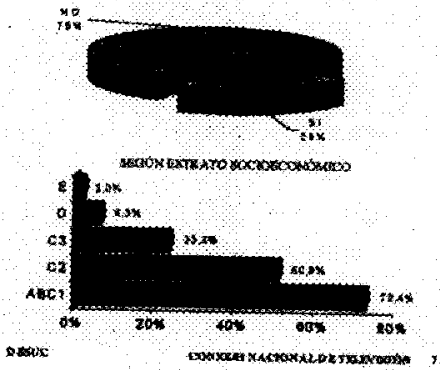


\section{COMUNICACION y MEDIOS}
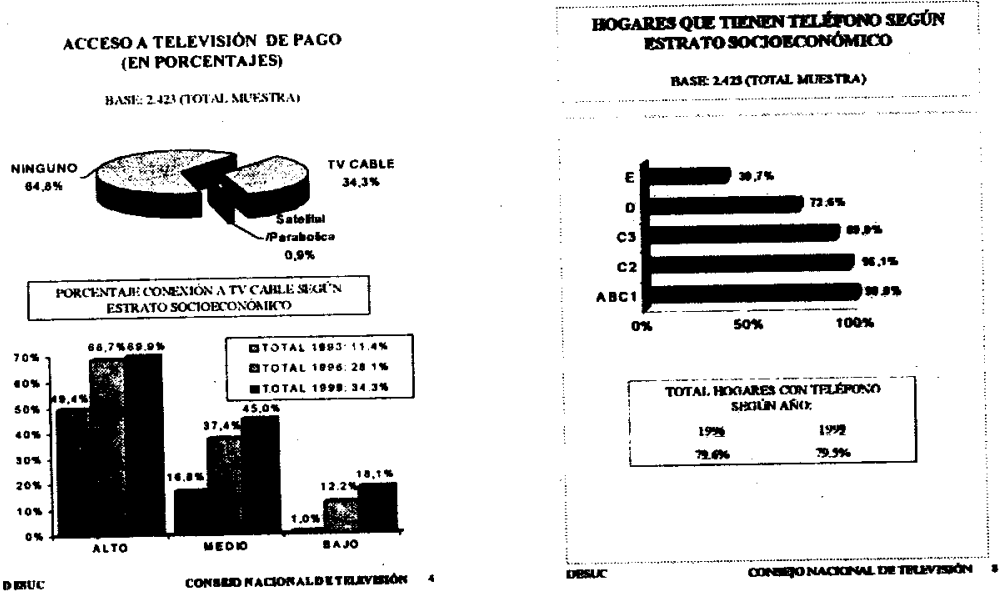

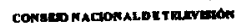

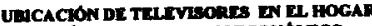 seotiN Estrato sociosconomico} (3 Mencionss)

BASE: HOQNRES QUE VEN TEIEVISION EN FAMR. IA (I.WO)

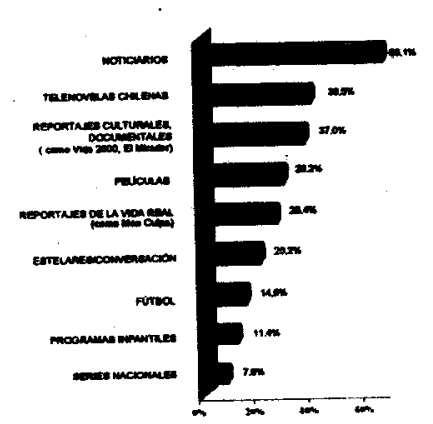

DEuc

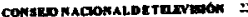

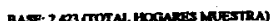

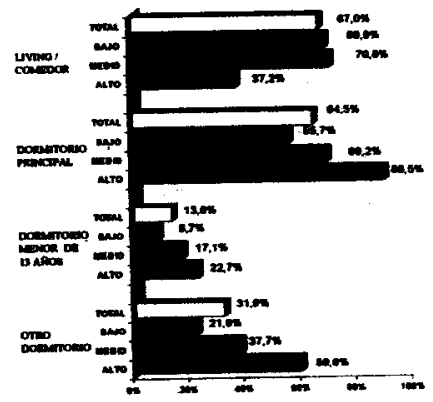

Dene

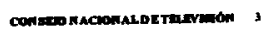


El equipamiento audiowisual hace factible la ausencia de referencia de un núdeo simbolico global lo que va a la parcon el aumento de la producción audiovisual, generándose, asi, la emergencia de microculturas, donde ya no hay un consumidor masivo sino que los integrantes de la familia tienen sus propias opciones de consumo.

Lo que se refeja en la ubicación de los televisores en el hogar, donde en los estratos altos sólo en un $89,5 \%$ esta en el dormitorio principal, y en los estratos medios en un $69,2 \%$; esto es, d consumo ya no es familiar sino que individual; sólo los noticseros tienen un alto consumo familiar un $66,1 \%$. Y cabra estudiar si realmente se ve o solo amuebla el espacio familia.

Esro significa que ha canbiado sustancialmente la ofera y el consumo cultural de medios, donde la dominante es hoy hausencia del consumidor masivo que fundamentó las bases de la propuesta del perfl del periodisa informador a crítico del sigto xx; y de todas las tecrologias periodísticas y pubiicicarias.

3. Categorias episténicas (prorocolos de lectura) presupuestos por las nuevas tecrologhas.

Lo anterior no sólosignifica la emergencia de un consumidor hipersogmentado que entra en ruptura con todo el saber de las comunicaciones respecto a la producción periodistica escrita, radial, o televisiva, basada en un consumidor masivo; sino que a través del control remoro y ha sobredosis de medias se genera e institucionalizará una cultura del zapping del fragmenro. Lo fragmental es constitutivo de esta nueva cultura, lo que no es una especulación teórica propuesta por Omar Calabrese en 1994, pues ello zene usa base empirica, en la misma realidad de consumo telexisivo dhileno (CNTV: Informe 1999).

El $60,3 \%$ ve un programa a la vez, frentea un $39,7 \%$ que mira varios en forma simultánea. El consumo fragmenndo, y el interpretar dos subconjuntas simultáneamente es propio de este nueva mirada caleidosó́pica frente a los medios. Es por ello que el ver televisión mientras se hace otras cosas es común al $51,5 \%$ de los consumidores. Lo que significa que las nociones de texro (ral como la entiende la linguistica del discurso o la semiótica tradicional), y las de su correlato periodístico (las nociones de crónica y de programa radial o televisivo) no rienen ningún sentido frente a esta nueva realidad del consumo cultural. Lo que el periodisca buscaba en generar una crónica o un programa radial o televisivo coherente, pues presuponia un consumo de un lectar atento que seguía paso a paso el desarrollo discursivo; lo que no tiene nada que ver con el consumo concreto de un $50 \%$ de la población. Lo mismo oaurte al semiólogo, analista de los mensajes culturales, pues el objeto de estudio ha variado consustancialmente ya no es cerrado sino que polidialogico. 
La lectura, entonces, es asociativa: mezda de fragmentos que se articulan con otra lógica que está en los link entre- textos, en la hipertextualidad. La hipertextualidad no es la cita de un texto dentro de otro texto como había sido descrita por Julia Kristeva en 1973 con el nombre de intertextualidad; sino que de argumentaciones paralelas. La hipertextualidad es esta competencia perceptiva, de a lo menos un $40 \%$ de la población del Gran Santiago de argumentar en forma paralela varios espacios cognitivos. El texto multimedial no es más que la expresión holográfica de una nueva propuesta no sólo de los formatos culturales, sino que tiene un correlato con pautas de consumo de las nuevas generaciones.

Las investigaciones sobre "el dibujo animado en Chile: sintaxis, circulación y recepción" de la unidad de estudio de la videoanimación (Proyecto Fondecyt No 1000954) de la Escuela de Periodismo de la Universidad de Chile avalan que las nuevas generaciones consumidoras de animación japonesa tienen un consumo fragmental e hipertextual que construye espacios perceptivos complejos que liga interconexiones de múltiples medios. Ramiro García y Cecilia Olmedo (2001), de dicha unidad de estudio, detectan que jugar Pokémon en el Games Boys es imposible de hacer con sólo las instrucciones del juego, sino que el consumidor requiere del saber de la serie televisiva, del saber de las revistas de comic (manga), y/o del saber dado a través de internet, y/o la película, y/o los videos del tema; esto es, se requiere de una competencia (un saber) de construir un sentido por medio de disímiles textos concretos. Lo mismo detectan respecto a la construcción de personajes en las series Dragón Ball, Dragón Ball Z, Dragón Ball GT, Card Captor Sakura, Evangelion, los investigadores Victor Fanjzylber, José Miguel Labrín, y Rafael del Villar de la misma unidad de estudio de la videoanimación de la Universidad de Chile.

Esto plantea la emergencia de estructuras complejas de significación que no tienen ninguna relación con los principios constitutivos de la pragmática comunicacional del periodismo y de la publicidad respecto a que la construcción de una información periodística y publicitaria debe ser simple, clara y sintética. Más bien, la fascinación de la videoanimación japonesa es el ser compleja, no simple ni evidente. Cabe destacar que la animación japonesa es preferida por los niños, en todos los estratos socioeconómicos, y se prefiere más que jugar, hacer deporte o ver programas deportivos (Del Villar, Fajnzylber, 2001).

$18 \mid$ Este desplazamiento de los espacios perceptivos imposibilitan y reiteran la imposibilidad de la noción de texto cerrado: de programa televisivo o radial autónomo, de artículo periodístico escrito y pensado como una unidad, una totalidad indivisible. 
Por otra parte, no es la única cransformación estatuida en la nueva episteme: los análisis de Christian Metz sobre el significante imaginario del cine que remitia al inconsciente psicoanaltico, continuados por Aumont; no sólo tienen una viabilidad en la microcultura del cine, sino que se expanden a la publicidad, instalando una propuesta onírica, pultsional, imaginaria en las sposs que la televisión nos propone. La descripción de una nueva propuesta publicitara que deja de lado la argumentación retórica lógica para introducinos en la ensoñación seductora es descrita ya por $\mathrm{Del}$ Villary por Gonzalez-Oriz desde $1922-95$ desde un punto de vista teórico. Sin embargo, ello no se trata sólo de una ensoñación reórica. La investigadora Erika Cortés detecta en un cotpus dé spot en televisión abierca, durante dos semanas, la existencia preponderance de h publicidad pulsionalo imaginaria " 3 in $60 \%$ de losespot son oniricos, lo gate esta en equitalencia a las nuevas propuestas teatrales que en un $70 \%$ tambitin bo som, y al $55 \%$ de los portadas de revista. Correlaciones similares se encuentron en el uss de lo fragmentado como sintaxis visual en el $56 \%$ en portadas de revistas, $67 \% \mathrm{~cm}$ nuevos tendencias teatrales, $y$ wn $62 \%$ de los espot".

Esto es, es evidente que nos encontramos con la emerzencia de nuevos protocolos inrerpretativos que entran en contradioción con el pensar una coherencia lógca argumentativa que tome a un texro, a un discurso como una unidad única, sino que mas bien se plantean espacios fragnentrados, oniricos de conexiones pluridialógicas.

Por otra parte, resulta evidente, tambien, que estos nuevos protocolos perceptivos propuestos por los medios no son comunes a toda la sociedad, sino que corresponden sólo a tn $40 \%$ de ella. La mayorla se enmanca (un 60\%) en la perspectiva tradicional de consumo de un texto cexrado.

E problema es que los presupuestos del quehacer semiótico sólo esca pensado en un $60 \%$ que sabernos que es solo una parte del constumidor, y que sabemos, además que ese otro $40 \%$ corresponde a las nuevas generaciones.

Situaciỏn similar se da en los palses desartollados, pero como lo avalan las investigaciones semióncas de François Jost sobre la televisión oomo vecror de identificación, los consumidores resemantizan la cultura global a partir de su propia realidad cultural.

\section{Pragmática comunicacional y semiótica}

Pero no son solo las nociones de texto y fragnento, de coherencia y de logica, las que son alteradas por esta nueva epistetne, tambien hay a lo menos las siguientes problemáticas que imposibilitan las competencias que se desarrollan hasta hoy instiacionalmente desde el punto de vista de la pragmática comunicacional y que necesizan de una semibtica que genere las herramientas para enfrentar estos nuevos pracesas: 
-el concepto de guión que se utiliza en la producción de espot publicitarios y programas televisivos está directamente ligado al establecimiento de una lógica narrativa de personajes que no tiene ninguna relación con los productos culturales oníricos desarrollados, habiendo un claro desequilibrio entre tecnologías de producción audiovisual y productos, lo que se ve reflejada en la comunicación ruidosa entre agencia de publicidad y productora de televisión, entre producción de programas periodísticos televisivos y los productos concretos que se generan;

-el concepto de guión multimedial utilizado tanto en la construcción de web como de periodismo informático saca su coherencia argumental, sus mapas de navegación como la estructuración de sus páginas, del cine narrativo clásico, al igual que el caso anterior, donde prima el conflicto- desarrollo-final, por sobre la oniricidad, y la fragmentación polidialógica e interconexión perceptiva multimedial, característica de las nuevas propuestas de consumo;

- La pirámide invertida de la tecnología periodística en uso corresponde al tratamiento de la noticia en sólo un espacio perceptivo, lo que no es equivalente a la multiplicidad códiga con que se transmite la información, tal como lo demuestran los estudios del investigador Eduardo Román;

-la producción y la programación tanto de radio como de televisión no se corresponde al consumidor textual presupuesto, sino que debiese construir con un presupuesto de ése otro lector descrito;

-la educación se ve enfrentada a nuevos procesos epistémicos de los consumidores culturales más jóvenes, lo que no dice relación con la retórica argumentativa de los textos y planes de enseñanza.

\section{Semiótica y Micro culturas}

Lo propio de esta nueva realidad emergente, no es sólo la emergencia de pérdida de un marco de referencia global (la mutación de un consumidor masivo a uno hipersegmentado), ni la emergencia de nuevas categorías episté-micas de producción y procesamiento de la información de los consumidores (en coexistencia con las antiguas), sino que la generación de microculturas.

Esto es; no sólo los textos (linguísticos, visuales, y audiovisuales) se consumen e interpretan en forma fragmentada, contradictoria, e hipertextualmente (no

20 | son textos cerrados, sino que su interpretación concreta remite a otros textos de diferentes soportes y campos culturales y/o ideológicos que cada subconjunto hace suyo), sino que implican la inserción de sus consumidores (a lo menos en el caso chileno) en toda una red de interacciones y rituales que permiten hablar de la emergencia de subculturas. 
Tal es el caso, entre ocros, de las siguientes microculnuras:

La literatura, que deja de ser un centro de referencia de la sociedad global (expresión de la cultura e idencidad chilena) para transformarse en un subconjunto cultural a la interior de la cultura global, lo que implica, a su vez, rupruras generacionales concretas. Dicha descripción hipotetica, quizas, es avalada por las estadisticas respecto al constmo no masivo de libros, la emergencia de talleres literarios, de congresos literarios, de actridiades literarias, y de suplementos literarios que sólo implican a sus consumidores participes de este tipo de manifestación cultural en toda una red de interacciones al respecto, que va desde lo pasivo (la cultura tradicionalliteraria) a lo activo (talleres, actos poeticos, etc). Es pasible tener la hipótesis de que las redes de interacción no son comunes a toda la microcultura, sino que ella es cortada por stbconjuntos constituidos generacionalmente, como es el caso de los suplementos "Revista de Libros" " y sección literatura de "Artes y lemas", ditigidos a la cultura literaria tradicional, y el suplemento "Zona de Contacto", referido a las microcultusas más jovenes, todos ellos ded diario "El Meraurio";

- dhip-hop, quien no es sólo un movimiento musical, sino que implica un espacio social para la criticz cultural, un espacio social para la realización de conciertos (muchas veces al aire libre) realizados más o menos espontáneamente por las comunas mismas, un espacio social para el desarrollo del ante del graffit, un espacio de relaciones sociales (amigos, contactos, etc), y un espacio de baile y danza. Sus sujeros se mueven en dichos espacios, tal como lo señala la investigacionn ("Hip-hop en Chile") sinterizada en esta revista, construyendo efectivamente una microculura;

-l graffit, puode ser entendido como un espacio aparte de la microculnurs del hip hop. Si bien es ciero que ambos espacios culturales se tocan en un punto, en lo de los graffiteros hip-hop, el graffiti en si es un espacio propio, es el espacio de expresión de "otra politica distinta a la politica tradicional". Uno de los aportes dela investigación "' Graffiti, espacio social y politica", sintetizada en este númeto, es describir como los objetos, los remas de estas expresiones culturales son referidas a "una micropoltica de la vida cotidiana", que haoe suya a las paredes de las calles para manifestar su diferencia con la politica del espacio de los politicos, implicando redes de acción referidas a comunidades territoriales, que encuentran en el graffiti una forma deser;

La video-aninación, no es sólo una referencia a rextos culturales como los video- juegos, el comic y/o manga, las seriales televisivas y los filmes de dibujos animados, los CD Ram, d lnternet; etc; sino que implica la emengencia de toda una serie de retaciones sociales a ello asociado, como lo es el intercambio de información en los colegios, sino que toda una red de interacciones de compra, arriendo, e inter- 


\section{COMUNICACION y MEDIOS}

cambio de saberes, que se da en Galerías Comerciales (Portal Lyon, Eurocentro, Persa Bío- Bío, en Santiago); que se han constituido en Espacios Sociales de esta Nueva Microcultura, donde el Gusto por la Animación Japonesa (Japoanimación) es preponderante, a lo que se suman, en Santiago el Cine Novedades que se trasforma no sólo en un tipo de cine que exhibe filmes japoneses de animación; sino que se trasforma en otro centro de reunión y de relaciones sociales de esta microcultura, tal como lo señalan, las investigaciones "Videoanimación en Chile: imagen, globalización y microculturas", y "Análisis semiótico comparativo Dragón Ball / Los Simpsons", sintetizadas en este número de la revista;

-lo étnico, y concretamente la cultura mapuche, participa de este proceso de constitución de espacios sociales microculturales, implicando toda una red de relaciones sociales e interacción con la sociedad blanca, a partir no de un todo homogéneo, sino que por sub-conjuntos étnicos, como lo señalan las investigaciones sintetizadas en este número: "Así son, así somos: un texto, dos lecturas, en la construcción de identidad", y"El disaurso público mapuche: complejo textual polisistémico producido para la prensa", La característica en común de estas microculturas (que existen entre otras, al interior de la realidad chilena) es su carácter no homogéneas. Lo que los artíaulos de las investigaciones sintetizadas en este número nos dicen, es que operan como universos contradictorios, polidialógicos, interrelacionando hasta disímiles puntos de vista.

Aproximándose a esta realidad de emergencias de subculturas, y a estos principios de funcionamientos fragmentados, contradictorios, de textos abiertos con relaciones hipertextuales complejas para su interpretación, y oníricos, surge toda una gama de manifestaciones culturales, que no constituyen una microcultura en sentido estricto, pues no implican una red de relaciones sociales ni rituales de interacción específicos entre sus usuarios, pero que síllevan la huella de estas nuevas formas de manifestación cultural: tal es el caso de la televisión para las nuevas generaciones, que constituyen su memoria histórica, la que va de lo global a lo local a través de la resemantización propia de sus consumidores culturales, como lo demuestra la investigación sintetizada "La televisión como vector de identidad"; y tal es el caso de las Nuevas Propuestas Teatrales Chilenas, que llevan la marca de esta intertextualidad entre publicidad, diseño gráfico y teatro, lo que se describe en la investigación sintetizada en este número "Funcionamiento intertextual entre nuevas propuestas teatra-

22 | les chilenas y publicidad, tanto televisiva como piezas de diseño gráfico".

\section{Construcción de Nuevas Identidades}

El sentido del III Encuentro Chileno de Semiótica, y de éste número de 
nuestra nevistz que sintetiza los principios construtwos del dossier de dicho evento, no tiene solo por función describir estas nuevas formas de funcionamiento cultural, sino que dar las bases para la inteligibilización de las nuevos procesos de construcción de identidades que de all ennergen.

No esperamos resolver el problema, sino que más bien el objetivo es contrbuir a su inteligibilizacion. Es claro que podrian ponerse en acto diferentes perspectrvas analiticas.

Pero, lo que es más daro, para quién escribe estas líneas (y organiza el dossiex) es que plantean nuevas formas de constnuoción de identidades, tanto a nivel micro-sacial, como macro.

Las conclusiones que se desprenden de las descripciones (sintetizadas como artculos en la presente revista) del hip hop, de la japonnimación, y de los textos énicos mapuches, es que no operan como una estructura homogénea, sino que contradicroriamente: no existe un solo principio constiturivo a la base. El caso del Hip- Hop es significativamente holográfico: de las entrevistas realizadas a participantes de esta microcultura se deduce que la identificación es poligonal. Esto es, a veces la identidad es ser D), a veces ser un participante de los lugares de encuentro (campeonatcs, tocatas, batallas), a veces es sólo una expansión en vias a sentirse participando de una comunidad; etc. Lo que significa que la identificación no opera como una cosmovisión coherente (un espacio para la crítica cultural, por ejemplo), tampoco, entonces, como una sola fuerza de identificación.

En el setrido lacanano y freudiano, la identificación opers conto "un ideal del yo", un deber ser que prefigura al sujeto a asumir un rol, homblogo a la ley simbólica reproductiva del orden social, osu inverso (un ideal contra fóbico). La idencificación serfa del orden de las sínbolos, de la idea, de la cosmovisión en definitiva.

Nada de esto ocurre en el Hip-Hop, ni en la Japoanimación.

El sujeto constnye y reconstruye su identidad a partir de una interrelacion entre diferentes complejos osub-connjunros.

En la Japoanimación, sus consumidores no se identifican con personajes, ni con ideas, ni cosmovisiones, sino que tratan de inteligibilizar lo real, para, por transacción reciproca entre distintos complejos constituir stu identidad, siempre por hacesse, por construirse. Nada parecido en ambos, a la identificacion secundaria de Lacan, Kristeva y Aumont.

Los nuevos procesos identificatorios son contradictorios, no homogéneos, pluridimensionales (polgonos), y muchas veces oníricos (primarios). Lo mismo ocurre en la identidad mapuche, lo que nos dicen ambas inveriga-ciones sintetizadas 
aquí, es que se da un sincretismo cultural, que interrelaciona elementos blancos con propios, pero que se leen a partir de un todo complejo contradictorio. Tal es el caso del discurso fotográfico analizado, como el de la demanda mapuche en su discurso público. Es la gestación, creemos, de nuevos procesos identificatorios los que están en juego.

Lo que es común a estas tres micro-culturas, y que platea la necesidad de nuevas herramientas analíticas para enfrentar su inteligibilización.

Lo que dicen, también, estas páginas, a nivel macro-social, es que esta forma de funcionamiento, descrita en la realidad chilena, entra en ruptura epistémica con lo descrito por Manuel Castells en "Le pouvoir de l'identité" (1999).

Para Castells, siguiendo a Touraine, hay tres formas de construir una identidad: "la identidad legitimante, que es introducida por el poder social al fin de alcanzar y racionalizar su dominación sobre los actores sociales, la identidad resistencia que es producida por los actores que se encuentran en posiciones o condiciones desfavorizadas y/o estigmatizadas por la lógica dominante, $y$ la identidad-proyecto, que aparece cuando los actores sociales, sobre la base del material cultural del cual ellos disponen, cualquiera que sea, construyen una identidad nueva" (1999:18).

Si la identidad resistencia conduce a comunidades, la identidad proyecto sigue la línea de Touraine y es entendida como un proyecto de sociedad, en definitiva. Sí esta última no se da hoy, en nuestras sociedades en redes, Castells ve la emergencia de la identidad resistencia: "nosotros hemos, también obervado la emergencia de unia poderosa identificación resistencia" (Castells, 1999: 427), que ligándose a comunidades alrededor de valores tradicionales-Dios, la nación, la familia-, o contestatarios (como el movimiento feminista) establecen una propia identidad construida en oposición a la identidad legitimante. Ahora bien, la identidad resistencia será el germen para la construcción de los nuevos proyectos de identidades: "los nuevos proyectos identidades no parecen deber venir de la antigua identidad de la sociedad civil de la era industrial, sino del desarrollo de las identidades- resistencias" (Castells, 1999: 428).

Nada de esto aparece en los procesos descrito en las investigaciones sintetizadas en este número, los que obviamente son referidos a nuestra realidad chilena.

24 El Hip- Hop, los Movimientos Étnicos, la Japoanimación, la Literatura, en los procesos descritos, están lejos de construirse como identidades de resistencia: no se construyen con un vector identificatorio (como la presuposición de Castells de costruirse en oposición a la identidad legitimante); sino que se construyen en forma contradictoria, polidialógica, y sü proyecto no es más que el acontecimiento, redefinido 
continuamente por la historia de la pequefia y gran existencia. Cabria preguntarse, acerca de si existe o no ese discurso legitmante, ante lo cual enenge este alternativo: Más bien, pensanas hiporéricamente, que se tratala de una ruprura con el prinapio de comuricación publica prodominanre en la anterionidad, generándose una pluraidad infinita de posibilidades que la copunnura y la historia define y redefine, aproximándase más a la situación renacentista de los inscios de ha sociedad industrial.

Hipóresis posibles. El sentido de este dassier no es "una descripción coherente de los procesas emergentes", sino que plantear problematizaciones desde un punto de vista analition. No se trata, entonces, de una críica a Castells, a Touraine, o a Matrellart; sino que más bien hacer conciencia de la necesidad de nevisión de muchas de las categorias analiticas, a partir de los datos que sabemos; esto es, de las investigaciones que aquí se sintetixan, y que correspondieron a comunicaciones realizadas en el masco del HI Encuentro Chileno de Semiótica.

\section{Bibliografla}

AUMONT, J. (1990) "Limage", Raris, Ed Nathan (1992) Trad Cast. "La imagen", Barcelona, Ed Paidós (1997), El ojo interminabie, Ed Paidós, Barcelona (1998), AUMONT, I, MARIE, M. (1988) "Lanalyse des films", Paris, Ed Nathan. (1990) Trad. Cast. "Analisis del Filn", Barcelona, Ed Paidos. AUMONT,MARE, VERNET,BERGALA, A. (1985) "Esthetique du film", Paris, Ed, Nathan. (1987), Trad. Cast., "Esrétics del Cine", Barcelona, Ed.Paidós.

CALABRESE, O., (1987), El lengraje del arte, Barcelona, Ed Paidós. (1994), La ero

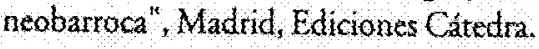

CARANI, M. (1998), Lyypoiconicité conme paradigne des atss visuels, Revue Visio Volume 3, Numero 1, Québec, Ed

CASTELUS (1998), "Societé en reseaux", Ed. Fayard, Paris. (1999), "Le pouvoir de 


\section{COMUNICACION y MEDIOS}

l'identité",Ed. Fayard, Paris; Trad. Portugés: (2000), "O poder da identidade",Ed. Paz e Terra, Sao Paulo. (1999), "Fin de millénaire", Ed. Fayard, Paris. (2001), "La Galaxie Internet",Ed. Fayard, Paris.

CATALÁN, C.; RAM, A. (1997), "Los cambios en la televisión chilena en los 90", en Revista Diálogos No 48, Ed. Felafacs, Lima.

CORTÉS, E... (1997), - "Relaciones Intertextuales entre Teatro y Danza", en CD Rom: Semiotics Bridging, International Association for Semiotics Studies, México 1997. (2000), "Funcionamiento intertextual entre nuevas propuestas teatrales chilenas y publicidad, tanto televisivas como de diseńo gráfico", en CD Rom "Sign Processes in Complex Systems", editado por Walter Schmitz; Ed. Technische Universitát Dresden, Alemania.

DEL VILLAR, R.. (1989) "Un outil sémiotique pour l'évaluation d'un vidéo educatif dans leur procès de gestation-production" en "Educational Development No 2". Seúl. Korea, Ed. Korean Educational Development Institute. (1992), "Una herramienta analítica audiovisual aplicada a la transmisión de la identidad latinoamericana" en Libro "En torno a la identidad latinoamericana" Méxiço, Ed. Felafacs (1997),"Trayectos en Semiótica Fílmica/ Televisiva", Santiago, Ediciones Dolmen. (1999), Nueva cultura audiovisual y protocolos interpretativos, En Libro: La pantalla delirante, editado por Carlos Ossa, Ed. LOM, Santiago. (1999), Desequilibrios de funcionamiento entre lo percibible y lo nombrable, en Revista Talón de aquiles, Ed. Uniacc, Santiago. (2000), "Videoculturas de fin de siglo: japoanimación, circuitos de circulación y resemantización local, en Revista Chilena de Semiótica No 4-5, Ed. Dept. Investigaciones Mediáticas y de la Comunicación, Facultad de Ciencias Sociales, Universidad de Chile. http://bibliotecas.uchile.cl/revistas/semiotica/ (2000), "Protocolos interpretativos empíricos de la japoanimación", en CD Rom "Sign Processes in Complex Systems", editado por Walter Schmitz; Ed. Technische Universitát Dresden, Alemania. (2000), "Videoculturas de la japoanimación", en Revista Comunicación y Medios, No 12, Año 12, Ed. Departamento de Investigaciones Mediáticas y de la Comunicación, Universidad de Chile, Santiago. (2001), "Videoanimación Americana y Japoanimación: categorías epistémicas presupuestas", en Revista Comunicación y Medios, No 13, Año 13, Ed. Departamento de Investigaciones Mediáticas y de la Comunicación, Universidad de Chile, Santiago DUBET, F.; WIEVIOKA, M. (1995; (Editores), "Penser le sujet. Autour d'Alain Touraine", Ed. Fayard, Paris.

ECO,U. (1968),"La struttura assente", Bompiani. (1974),Trad. Cast. "La estrucura

26 | ausente", Barcelona, Ed. Lumen. (1974), "¿El público perjudica a la televisión?, (1979), Trad. Casten Moragas, M.de, Editor, Sociología de la Comunicación de Masas, Barcelona, Ed. Gustavo Gili. (1979), "Lector in fabula", Bompiani. (1981), Trad. Cast. "Lector in fabula", Barcelona, Ed. Lumen. (1990) "I limiti dell'interpretazione", FabbriBompiani, (1992) Trad. Cast. "Los límites de la interpretación", Barcelona, Ed. Lumen. 
(1992) "Interpretation and overinterpretation", Cambridge, Cambridge University Press. (1995), Trad. Cass. "Ineerpretación y sobreinterpretación", Cambridge, Cambridge Universiry Press. (1998), Refiexions a propos du debar sur liconisme, en Revue Visio Volume 3, Numero 1, Quêtec, Ed Université du Laval.

FAJNZYLVER, V. (2000), El lenguaje de la videoanimación, en Revisa Chilena de Sensiótica No 4-5, Ed. Dept. Investigaciones Mediásicas y de la Comunicación, Facultad de Ciencias Sociales, Universidad de Chile. Interner: hrtp:/bibliotecas. uctille.d) revistas/semiorical (2001) "Videoanimación en Chile imagen, globalización y microculturas", en Revista Comunicación y Medios, No 13, Año 13, Ed Deparamento de Investigaciones Mediáticas y de la Comunicacion, Universidad de Chile, Santiago.

FERNANDEZ, MARTINEZ (1994), La dirección de praducción para cine y televisión, Ed. Paidós, Barcelona.

FERNÁNDEZ COCA, A. (1998), Producción y diseño gráfico para la Wordd Wide Web?, Ed. Paidos, Barcelona.

FLOCH, J M. (1990), Semiotique, Marketing et Conmmunication, Ed. PUF, Paris. (1995), Identités visuelles, Ed. PUF, Paris.

GONZALEZ \}, (1995), El discurso relevisivo: espectáculo de la posmodernidad, Madrid. Ed Cátedra.

GONZALEZZ J); ORTIZ A (1999), El espot publicitario. La metamorfósis del deseo, Madrid Ed Cátedra

GRANDI R. (1997), (Ediror), Semiotica al marketing, Milano, Ed FrancoAngeli. JOSEPH, 1. (1999), ?Erving goffman y la microsociologha?, Ed Gedisa, Barcelona. JOST F (1989), "L'oei-Caméra", Ed. PUL, Lyon (1997), La celétision aux frontières du réel, en Revue Esprit, Decembrre 1997. (1997), Présentation, en Revue Réseaux No 81, fevier 1997. (2000), Le contrar de communication une illusion fin de siecde, en Revista Signa, Actas IV Congreso Fedesación Latinoamericana de Semiótica, Madrid, (2001), "El género televisual del contrato a la promess", en Revista de Cine No 1, Fd. Facultad de Artes, Universidad de Chile (2001), "La television du quoridien", Ed. DeBoeck Université, Paris (2002), "La televisión como vector de identidad", en en Revisa Comunicación y Medios, No 13, Año 13, Ed Deparamento de Investigaciones Mediáticas y de la Comunicación, Universidad de Chile, Santiago

KRISTEVA, (1974), "La révolution du langage poétique", Paris, Ed. Du Seuil. (1975), "La traversee des signes". Paris, Ed. Du Seull (1977), "Polylogue", Paris, Ed. Du Seuil (1979), "Folle vertite", Paris, Ed Du Seull (1980), "Pouwoirs de l'horreur", Paris, Ed. Du Seuil. (1983), "Histoifes d'amour", Paris, Ed. Denoë. (1996), "Sens et non-sens de la révolte", Paris, Ed. Fayard. (1997), "La révolte inime", Paris, Ed. Fayard. (1998), "Contre la depression nationale", Paris, Ed Textuel. (2001), "Micropolitique", Ed, de l'aube; Paris.

LABRAN, 3. POBLETE, P, LETELIER, L, LECHUGA, L, (2000) Semítica del 
Dragón Ball Z, en Revista Chilena de Semiótica No 4-5, Ed. Dept. Investigaciones Mediáticas y de la Comunicación, Facultad de Ciencias Sociales, Universidad de Chile. Internet: http://bibliotecas.uchile.cl/revistas/semiotica/

LACAN,J., (1938), "La famille", Paris, Encyclopédie Francaise, Ed. A de Monzie. (1982), Trad. Cast., "La Familia", Barcelona, Ed. Argonauta. (1966) "Ecrits", Paris, Ed. du Seuil. (1973), "Les quatre concepts fondamentaux de la psychanalyse". Paris, Ed. du Seuil. (1981), "Les psychoses", Paris, Ed. du Seuil. (1993), Trad. Cast. "Las psicósis", Buenos Aires, Ed. Paidós.

LANDOW, G. (1992), ?Hypertext: The convergence of Contemporary Critical Theory and Technology, Baltimore, Johns Hopkins Ineversity Press. (1997), Teoría del hipertexto, Ed. Paidós, Barcelona

LAWANDOS, R. (2002), "Dibujo animado e impresión de realidad", en Revista Comunicación y medios No 13, año 13, Ed. Dept Investigaciones Mediáticas y de la Comunicación, y Escuela de Periodoismo, Universidad de Chile, Santiago.

LE MÉHAUTÉ, A. (1990), "Les géomérries fractales", Paris, Ed. Hermes.

LÉVI-STRAUSS, C. (1962),"La pensée sauvage",Paris, Ed.Plon. (1958), "Anthropologie Structurale", Paris, Ed. Plon. (1968), Trad. Cast. "Antropología Estructural", Buenos Aires, Ed.Eudeba. (1968), "Mitológicas: Lo crudo y lo cocido", México, Ed. Fondo de Cultura Económica. (1973),"Athropologie Structurale Deux", Paris,. Ed. Plon. (1979), "La voie des masques", Paris, Ed. Plon.

LÉVI- STRAUSS, C.; ERIBON. (1988), "De prés et de loin", Paris, Ed. Odile Jacob.

MANDELBROT, B. (1975), "Les objets fractals", Paris, Ed. Flammarion. (1997), "Fractales, hasard et finance", Paris, Ed. Flammarion. (1997), La geometría fractal de la naturaleza, Barcelona, Ed. Tusquets.

MARCUS, S. (1967), "Introduction mathématique à la linguistique structurale", Paris, Ed. Dunod.

MASOTA,O.(1977),"Lecciones de Introducción al Psicoanálisis", Barcelona, Ed. Gedisa.

MEDEL, I. (2000), "Semiótica del audiovisual: japoanimación y género", en Revista Chilena de Semiótica No 4-5, Ed. Dept. Investigaciones Mediáticas y de la Comunicación, Facultad de Ciencias Sociales, Universidad de Chile. Internet: http:// bibliotecas.uchile.cl/revistas/semiotical

METZ, CH. (1968), "Essais sur la signification au cinéma", Tomo 1, Ed. Klincksieck, Paris. Hay Trad. Español, Ed. Tiempo Contemporáneo, Buenos Aires, 1970. (1973),

28 "Essais sur la signification au cinéma", Tomo 2, Ed. Klincksieck, Paris. (1971), "Langage et cinéma", Ed. Larousse, Paris. (1977), "Essais Sémiotiques", Ed. Klincksieck, Paris. (1979),"Psicoanálisis y cine. El significante imaginario", Ed. Gustavo Gili,

MILLER D. (1997), Desarrollo multimedia para Internet, Madrid, Ed. Anaya. 
MITCHELL, J (1975), "\$sychanalyse et féminisme", Tono ly II, Paris, Ed Des Fernmes.

MOREIRA, H. (1994), "Cuerpo de rnujer", Montevideo, Ed Trilce.

THOM, R (1987), "Estabilidad estructural y morfogénesis", Barcelona, Editorial Gedisa (1990), "Esbozo de una semiofisiza", Barcelona, Editorial Gedisa. (1987), "Prédire n'est pad expliquer", Paris, Ed. Flanmarion. (1995), "Paraboles et cacastrophes", Pars, Ed. Elammation.

TROUT ], RIES A. (1981), Posicionamiento: la batalla por su mente, Madrid, Ed. Mc Graw Hill (1996), El ntevo posicionamiento, Madrid, Ed Mc Graw Hill UMIKER - SEBEOK (1987), Marketing and semiorics, Berlin, Ed. Mouron de Gruyter. (1994), "Setniótica del marketing en la Universidad de Indiana", Ed. Unversidad de Indiana, Indiana. Hay trad. Española.

VANOVE, $\mathrm{E}$ (1996), Guines modelo y modelos de guión, Ed. Paidós, Barcelona VARELA, E. (1989), "Autonomie er connaissance", Paris, Ed. du Seuil. (1996), "Conocer", Barcelona, Ediciones Gedisa

VARELA, F; THOMPSON, E; ROSCH, E. (1992), "De cuerpo presente", Barcelona, Ed. Gedisa.

VLCHES, L (1983), La lectura de la inagen, Ed. Paidós, Barcelona (1987), Teoria de la imagen periodistica, Ed. Pridós, Barcelona. (1989), Manipuación de la información televisiva, Ed Paidós, Barcelona. (1993), La televisión. Los efecros del bien y del mal, Ed. Paidos, Barcelona.

VILLAIN, D. (1997), El encuadre cinematográfico, Ed. Paidós, Barcelona.

VV. AA. (1996), "Videoculturas de fin de siglo", Madrid, Ed. Cátedra. WEYL, H. (1996), "Symétrie er mathémarique moderne", Paris, Ed. Flamnarion. WTTHALM, G. (2000), "When a film turns into a film in the making... Multiple stories and recursive loops",

en CD Rom "Sign Processes in Complex Systems", editado por Walter Schmitz; Ed. Technische Universitát Dresder, Alemania. 
